\title{
Alteration of maternal serum irisin levels in gestational diabetes mellitus
}

\author{
Ulku Mete Ural' ${ }^{1}$ Serap Baydur Sahin2 ${ }^{2}$ Yesim Bayoglu Tekin', \\ Medine Cumhur Cüre ${ }^{3}$, Hacer Sezgin ${ }^{4}$ \\ ${ }^{1}$ Department of Gynecology and Obstetrics, Recep Tayyip Erdogan University Medical Faculty, Rize, Turkey \\ ${ }^{2}$ Department of Endocrinology and Metabolic Diseases, Recep Tayyip Erdogan University Medical Faculty, Rize, Turkey \\ ${ }^{3}$ Department of Biochemistry, Recep Tayyip Erdogan University Medical Faculty, Rize, Turkey \\ ${ }^{4}$ Department of Family Medicine, Recep Tayyip Erdogan University Medical Faculty, Rize, Turkey
}

\begin{abstract}
Objectives: The aim of our study was to compare serum irisin concentrations in pregnant women with and without gestational diabetes mellitus (GDM).

Material and methods: This study was performed at the Tertiary Care Center, Department of Obstetrics and Gynecology, between January 2014 and April 2014. A total of 45 pregnant women with GDM (diabetes group) and 41 BMI- and age-matched healthy pregnant women (control group) were recruited. Maternal serum irisin levels were measured by enzyme-linked immunosorbent assay kit at 24-28 weeks of gestation. An association between maternal serum irisin levels and metabolic parameters was analyzed. Body mass index, serum levels of glucose, insulin and irisin were tested and analyzed in the study group and controls.

Results: Pregnant women with GDM had significantly higher fasting plasma glucose $(p=0.001)$, first-hour OGTT glucose $(p=0.001)$, second-hour OGTT glucose $(p=0.001)$, and fasting insulin $(p=0.045)$ levels as compared to controls. Serum irisin levels were $1.04 \pm 0.3$ and $1.3 \pm 0.2$ in pregnant women with GDM and healthy pregnant controls, respectively $(p=0.001)$ Correlation analysis between irisin levels and anthropometric and biochemical parameters in patients with gestational diabetes revealed that none of the investigated parameters correlated with serum irisin level.

Conclusions: Our results suggest that serum irisin levels might be introduced as a novel marker for GDM, with decreased levels of irisin being indicative of GDM.
\end{abstract}

Key words: irisin, pregnant, gestational diabetes mellitus, glucose, insulin

Ginekologia Polska 2016; 87, 5: 395-398

\section{INTRODUCTION}

Gestational diabetes mellitus (GDM) is defined as a carbohydrate intolerance of varying severity, with onset or first recognition during pregnancy. GDM prevalence may range from $1 \%$ to $14 \%$ of all pregnancies, depending on the studied population and the diagnostic test employed [1]. The pathogenesis of GDM is multifactorial and may include genetic and environmental factors, but the exact mechanism remains to be fully elucidated [2]. Women with GDM are at increased risk of perinatal morbidity, impaired glucose tolerance, and type 2 diabetes in the years after pregnancy. Children born to women with GDM are also more likely to be obese, have impaired glucose tolerance and diabetes in childhood and early adulthood [3].

In the past few years, dysregulation of various adipocyte- and hepatocyte-derived factors (including adiponectin, leptin, fibroblast growth factor-21, and adipocyte fatty acid-binding protein) has been reported to mediate insulin resistance and proinflammatory effects in GDM [4, 5]. Moreover, myocyte-secreted proteins, in addition to adipokines and hepatokines, generated renewed interest in the field of metabolic diseases most recently. In this context, the fundamental study of Boström et al., introduced the myokine irisin as an exercise-inducible secreted factor 
that improves glucose tolerance and increases energy expenditure in mice [6].

So far, data about irisin in humans have been insufficient to evaluate its metabolic effects and association with metabolic disease. Irisin is an anti-diabetic hormone that regulates the glucose metabolism and energy consumption via converting white to brown adipose tissue [7]. Reports on irisin regulation in GDM and pregnancy are scant.

\section{OBJECTIVE}

To the best of our knowledge, only five studies on the association between irisin and GDM have been reported in the literature [8-12]. In light of the conflicting results of these studies, we also wished to investigate this topic. The aim of our study was to compare serum irisin concentrations between pregnant women with GDM and healthy pregnant women. Serum irisin levels may have a potential as a novel marker for diagnosis and follow-up of gestational diabetes mellitus.

\section{MATERIAL AND METHODS}

This study was approved by the Local Ethics Committee (no: 2014/64) and written informed consent was obtained from all subjects. A total of 45 pregnant women with gestational diabetes mellitus (diabetes group) and $41 \mathrm{BMI}$ - and age-matched healthy pregnant women (control group), admitted to our tertiary care center, Department of Obstetrics and Gynecology between January 2014 and April 2014, were recruited for this case-control study. Hypertensive pregnant subjects, patients $>40$ years of age, and pregnant women with $\mathrm{BMI}>30$ were excluded from the study.

All participants underwent a thorough clinical examination. The diagnosis of GDM was based on the International Diabetes in Pregnancy Consensus Group criteria [13]. The diagnosis of GDM was made according to the HAPO criteria, when one of the following plasma glucose values in the oral glucose tolerance test (OGTT) was met or exceeded: fasting plasma glucose $92 \mathrm{mg} / \mathrm{dL}$ (5.1 mmol/L); 1-h plasma glucose $180 \mathrm{mg} / \mathrm{dL}$ (10.0 mmol/L); and 2-h plasma glucose $153 \mathrm{mg} / \mathrm{dL}$ ( $8.5 \mathrm{mmol} / \mathrm{L})$. Body mass index was determined as weight divided by squared height. Irisin serum concentrations were determined with an enzyme-linked immunosorbent assay (Human Irisin ELISA kit, MyBiosource Inc, San Diego, CA, USA) according to the manufacturer's instructions at 24-28 gestational weeks. Fasting serum irisin level was measured 3 days after the OGTT test. Furthermore, fasting insulin was determined with a two-site chemiluminescent enzyme immunometric assay. Moreover, glycosylated hemoglobin $\mathrm{A}_{1 c}\left(\mathrm{HbA}_{1 c}\right)$ was measured. The association between maternal serum irisin levels and metabolic parameters was analyzed.

\section{Statistical analysis}

Data were analyzed using the Statistical Package for Social Sciences 19.0 for Windows (SPSS Inc., Chicago, IL). Data were tested for normality with the Shapiro-Wilk test. Data with a normal distribution (blood pressure values, serum glucose, insulin, and irisin levels) were compared with the student's t test (correlation coefficients were calculated with Pearson's r), and expressed as mean \pm SD. Data without normal distribution (age, BMI) were compared with the Mann-Whitney-U test, and expressed as median (interquartile range). The $p$-value of $<0.05$ was considered as statistically significant.

\section{RESULTS}

A total of 84 pregnant women met the eligibility criteria for the study. The diabetes group included 45 pregnant women with gestational diabetes mellitus and the control group included 41 of their age-matched healthy pregnant peers.

Both groups did not differ in terms of age $(p=0.759)$, BMI $(p=0.743)$, weight gain $(p=0.354)$, systolic $(p=0.665)$, and diastolic blood pressure $(p=0.789)$. Pregnant women with GDM had significantly higher fasting plasma glucose ( $p=0.001)$, first-hour OGTT glucose level $(p=0.001)$, second-hour OGTT glucose level ( $p=0.001)$, and fasting insulin $(p=0.045)$ levels as compared to the control group. Serum irisin level was $1.04 \pm 0.3$ in pregnant women with GDM, and $1.3 \pm 0.2$ in controls $(p=0.001)$ (Figure 1 , Table 1 ).

Correlation analysis between irisin levels and anthropometric and biochemical parameters in patients with gestational diabetes revealed none of the parameters to be correlated with serum irisin level (Table 2).

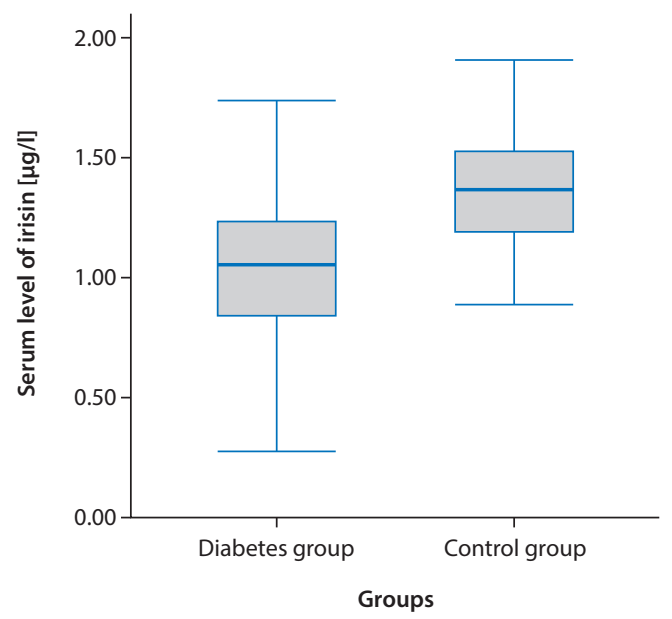

Figure 1. Serum irisin level in pregnant women with GDM and in controls 
Table 1. Comparison of clinical and biochemical results between the patients with gestational diabetes mellitus and control subjects

\begin{tabular}{|c|c|c|c|}
\hline Variable & $\begin{array}{l}\text { Diabetes group } \\
\qquad(n=45)\end{array}$ & $\begin{array}{l}\text { Control group } \\
\qquad(n=41)\end{array}$ & $p$ value \\
\hline Age (years) & $30(26-34)$ & $29(26-33)$ & 0.759 \\
\hline BMI at the time of OGTT $\left[\mathrm{kg} / \mathrm{m}^{2}\right]$ & $28.6(24.2-31.6)$ & $26.8(23.6-31.5)$ & 0.743 \\
\hline Weight gain [kg] & $7.4 \pm 3.9$ & $8.2 \pm 4$ & 0.354 \\
\hline Systolic blood pressure[mm Hg] & $114.3 \pm 12.5$ & $113 \pm 14.5$ & 0.665 \\
\hline Diastolic blood pressure [mm Hg] & $70.3 \pm 9.1$ & $70.8 \pm 8.9$ & 0.789 \\
\hline Fasting plasma glucose $[\mathrm{mg} / \mathrm{dL}]$ & $94.3 \pm 10.2$ & $79.9 \pm 4.8$ & $0.001^{*}$ \\
\hline OGTT $-1^{\text {st }}$ hour plasma glucose $[\mathrm{mg} / \mathrm{dL}]$ & $190.2 \pm 29.8$ & $117.1 \pm 20.1$ & $0.001^{*}$ \\
\hline OGTT $-2^{\text {nd }}$ hour plasma glucose $[\mathrm{mg} / \mathrm{dL}]$ & $142.9 \pm 37.2$ & $101.2 \pm 14.6$ & $0.001^{*}$ \\
\hline Fasting insulin $[\mu \mathrm{lU} / \mathrm{mL}]$ & $9.2 \pm 3.7$ & $7.5 \pm 3.9$ & $0.045^{*}$ \\
\hline Irisin & $1.04 \pm 0.3$ & $1.3 \pm 0.2$ & $0.001^{*}$ \\
\hline
\end{tabular}

*Statistically significant. BMI — body mass index; OGTT — oral glucose tolerance test

Table 2. The correlation between irisin levels and anthropometric and biochemical parameters in patients with gestastional diabetes

\begin{tabular}{|l|c|c|}
\hline Parameter & $\mathbf{r}$ & $\mathbf{p}$ \\
\hline Age & -0.26 & 0.071 \\
\hline Weight at the time of OGTT & -0.030 & 0.844 \\
\hline BMI at the time of OGTT & -0.038 & 0.801 \\
\hline Weight gain & 0.098 & 0.513 \\
\hline Systolic blood pressure & 0.045 & 0.766 \\
\hline Diastolic blood pressure & 0.196 & 0.186 \\
\hline Fasting plasma glucose & 0.084 & 0.577 \\
\hline OGTT - 15t hour plasma glucose & 0.022 & 0.882 \\
\hline OGTT - 2 ${ }^{\text {nd }}$ hour plasma glucose & -0.132 & 0.378 \\
\hline Fasting insulin & -0.141 & 0.344 \\
\hline
\end{tabular}

\section{DISCUSSION}

In this study, we attempted to compare serum irisin concentrations between pregnant women with GDM and age-matched healthy pregnant women, and found that maternal serum irisin levels were significantly lower in pregnant women with GDM as compared to controls. Recently, a muscle-secreted peptide named irisin was identified and suggested to play an important role in improving obesity and glucose homeostasis [14]. Irisin has attracted considerable attention because it has been claimed to have therapeutic potential for obesity and diabetes and to improve life expectancy [15].

A positive association between circulating irisin and markers of glucose intolerance was detected by two independent studies in non-pregnant subjects $[16,17]$. Huh et al., showed that irisin correlated positively with fasting glucose in 117 healthy non-pregnant women [16]. Stengel et al., demonstrated that circulating plasma irisin is posi- tively associated with fasting insulin level [17]. In contrast, Moreno-Navarrete et al., showed that the expression of the irisin encoding gene FNDC5 in adipose and muscle tissue is reduced in obese and diabetic patients as compared to non-obese subjects [18]. Furthermore, irisin serum levels are lower in Chinese patients with new-onset T2DM as compared to non-diabetic subjects [18]. Two previous reports observed a decreased level of circulating irisin in patients with type 2 diabetes as compared to normal glucose tolerance subjects [18-20]. All things considered, the current and the previous results support the hypothesis that increased circulating irisin is an adaptive response to compensate for the decreasing insulin sensitivity and disturbances in metabolism associated with obesity [21]. Although irisin is also secreted by adipose tissue, the reduced irisin levels displayed in type 2 diabetes may be due to the decrease of body fat stores in uncontrolled insulin-deficient diabetes as is the case with leptin [22]. In our study, serum irisin level was lower in pregnant women with GDM as compared to healthy pregnant women.

The relationship between irisin and markers of energy metabolism has been explored earlier by other authors. Similarly to our results, Timmons et al., reported that irisin was not related to markers of energy metabolism including BMI, fasting insulin, and fasting glucose [23]. However, Huh et al., found that circulating irisin concentrations were positively correlated with BMI and fasting glucose [16]. We are of the opinion that these discrepancies may be explained by the differences in their study population. Timmon et al., analyzed the association between myocyte irisin and markers of energy metabolism in a diabetic population, while Huh et al., conducted their study in healthy women [23]. Kuzmicki et al., stated that serum irisin concentration increased remarkably in pregnant women, but this increase was significantly less obvious in patients with GDM [9]. In our study, a correlation 
analysis of irisin levels and anthropometric and biochemical parameters in patients with gestational diabetes revealed that none of the parameters correlated with serum irisin level.

Limitations of our study included a relatively small sample size, and lack of definitive selection criteria for the BMI. Although BMI level was not different between the groups, we had patients with the BMI below 25 or above 30 . Also, we did not include serum level of the lipid profile due to the lack of data in most of the investigated patients. In light of these restrictions, associations should be interpreted with caution.

\section{CONCLUSIONS}

Maternal serum irisin levels were significantly lower in pregnant women with GDM as compared to the control group. This result suggests that serum irisin levels might be introduced as a novel marker for GDM and decreased levels of irisin might be indicative of GDM. Further randomized, prospective, controlled trials on larger series are necessary to make more precise interpretations.

\section{Conflict of interests}

The authors declare no competing interest. No financial support was received for this paper.

\section{REFERENCES}

1. American Diabetes Association. Gestational diabetes mellitus. Diabetes Care. 2004, 27 (1), 88-90.

2. Metzger BE, Coustan DR. The Organizing Committee: summary and recommendations of the Fourth International Workshop-Conference on Gestational Diabetes Mellitus. Diabetes Care. 1998, 21 (2), 161-167.

3. Zhang C, Ning Y. Effect of dietary and lifestyle factors on the risk of gestational diabetes: review of epidemiologic evidence. Am J Clin Nutr. 2011, 94 (6), 1975-1979.

4. Kralisch S, Stepan H, Kratzsch J, [et al.]. Serum levels of adipocyte fatty acid binding protein are increased in gestational diabetes mellitus. Eur J Endocrinol. 2009, 160 (1), 33-38.

5. Miehle K, Stepan H, Fasshauer M. Leptin, adiponectin and other adipokines in gestational diabetes mellitus and pre-eclampsia. Clin Endocrinol. 2012, 76 (1), 2-11.
6. Boström P,Wu J, Jedrychowski MP, [et al.]. A PGC1-a-dependent myokine that drives brown-fat-like development of white fat and thermogenesis. Nature. 2012, 481 (7382), 463-468.

7. Aydin S. Three new players in energy regulation: preptin, adropin and irisin. Peptides. 2014, 56, 94-110.

8. Ebert T, Stepan H, Schrey S, [et al.]. Serum levels of irisin in gestational diabetes mellitus during pregnancy and after delivery. Cytokine. 2014, 65 (2), 153-158.

9. Kuzmicki M, Telejko B, Lipinska D, [et al.]. Serum irisin concentration in women with gestational diabetes. Gynecol Endocrinol. 2014, 30 (9), 636-639.

10. Yuksel MA, Oncul M, Tuten A, [et al.]. Maternal serum and fetal cord blood irisin levels in gestational diabetes mellitus. Diabetes Res Clin Pract. 2014, 104 (1), 171-175.

11. Piya MK, Harte AL, Sivakumar K, [et al.]. The identification of irisin in human cerebrospinal fluid: influence of adiposity, metabolic markers, and gestational diabetes. Am JPhysiol Endocrinol Metab. 2014, 306 (5), 512-518.

12. Aydin S, Kuloglu T, Aydin S. Copeptin, adropin and irisin concentrations in breast milk and plasma of healthy women and those with gestational diabetes mellitus. Peptides. 2013, 47, 66-70.

13. International Association of Diabetes and Pregnancy Study Groups Consensus Panel, Metzger BE, Gabbe SG, Persson B, [et al.]. International association of diabetes and pregnancy study groups recommendations on the diagnosis and classification of hyperglycemia in pregnancy. Diabetes Care. 2010, 33 (3), 676-682.

14. Hofmann T, Elbelt $U$, Stengel A. Irisin as a muscle-derived hormone stimulating thermogenesis - a critical update. Peptides. 2014, 54, 89-100.

15. Sanchis-Gomar F, Lippi G, Mayero S, [et al.]. Irisin: a new potential hormonal target for obesity and type 2 diabetes. J Diabetes. 2012, 4 (3), 196.

16. Huh JY, Panagiotou G, Mougios V, [et al.]. FNDC5 and irisin in humans: I. Predictors of circulating concentrations in serum and plasma and II. mRNA expression and circulating concentrations in response to weight loss and exercise. Metabolism. 2012, 61 (12), 1725-1738.

17. Stengel A, Hofmann T, Goebel-Stengel M, [et al.]. Circulating levels of irisin in patients with anorexia nervosa and different stages of obesity — correlation with body mass index. Peptides. 2013, 39, 125-130.

18. Moreno-Navarrete JM, Ortega F, Serrano M, [et al.]. Irisin is expressed and produced by human muscle and adipose tissue in association with obesity and insulin resistance. J Clin Endocrinol Metab. 2013, 98 (4), 769-778.

19. Choi Y-K, Kim M-K, Bae KH, [et al.]. Serum irisin levels in new-onset type 2 diabetes. Diabet Res Clin Pract. 2013, 100 (1), 96-101.

20. Liu JJ, Wong MD, Toy WC, [et al.]. Lower circulating irisin is associated with type 2 diabetes mellitus. J Diabetes Complications. 2013, 27 (4), 365-369.

21. Hojlund K, Bostrom P. Irisin in obesity and type 2 diabetes. $J$ Diabetes Complications. 2013, 27 (4), 303-304.

22. Mohammadzadeh G, Zarghami N. Serum leptin level is reduced in non-obese subjects with type 2 diabetes. Int J Endocrinol Metab. 2013, 11 (1), 3-10.

23. Timmons JA, Baar K, Davidsen PK, [et al.]. Is irisin a human exercise gene? Nature. 2012, 488 (7413), 9-10. 